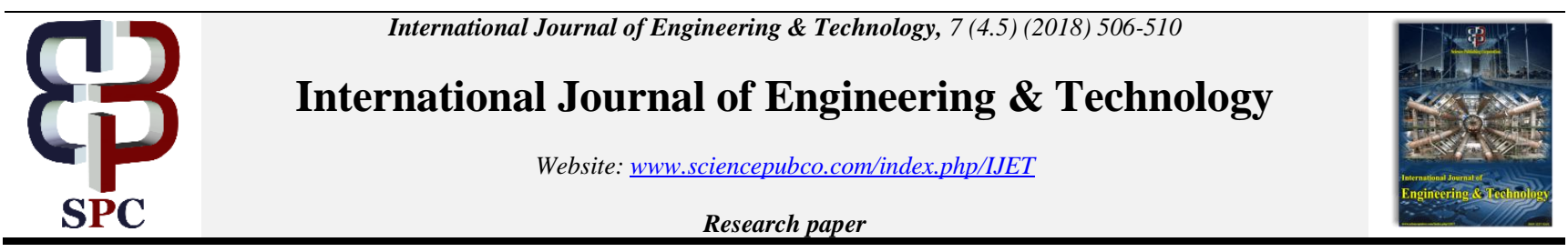

\title{
A survey on vision based techniques for detection and classification of fruit diseases
}

\author{
VipponPreet Kour ${ }^{1}$, Sakshi Arora ${ }^{1}$, Jasvinder Pal Singh ${ }^{1}$ \\ ${ }^{1}$ Department of Computer Science \&Engineering, SMVDU, Katra, Jammu, India \\ *Corresponding author E-mail: sakshi@smvdu.ac.in
}

\begin{abstract}
Indian economy depends heavily on its agriculture system. Horticulture sector has a major share in Indian agrarian economy. Therefore for agriculture industry to grow, the effective growth and improved yield of fruits is necessary. Diseased fruit production lays down various sensitive issues that can create various crises including reduced exports. To tackle such situations farmers need manual monitoring of fruits in all the development phases till harvest. But manual monitoring may not always give satisfactory results, owing to the subjective nature of the process. In order to reduce this stress, the technological support for such monitoring of fruit diseases was introduced. Image processing is one of the widely accepted areas for fruit disease detection and classification. With accurate disease diagnosis, the proper control actions can be taken at appropriate time. This paper is intended to aid in the analysis of various techniques and methodologies used in fruit disease detection so far.
\end{abstract}

Keywords: Image Processing; Image Segmentation; Image Acquisition; Training and Classification.

\section{Introduction}

The agriculture is more than just a feeding area these days. A fine amount of country's economy is dependent on the agriculture. With such a value, this area needs consideration for development and production. According to certain scientific analysis, the demand for food and the production ratio is going to be 80:30 approximately in the upcoming years. So in order to deal with such situations, the more technical and focused approach should be implemented for the production. Agronomy is a combination of various branches e.g., aeroponic, geoponic, hydroponic as shown in figure 1.These branches include different sub-branches which include all technical and non-technical fields of agriculture. Horticulture comes under the geoponic category. This area is widely followed all over the world anddue to frequent climatic changes; this area is also prone to many diseases. Therefore, to detect the diseases, automatic disease detection techniques are beneficial.

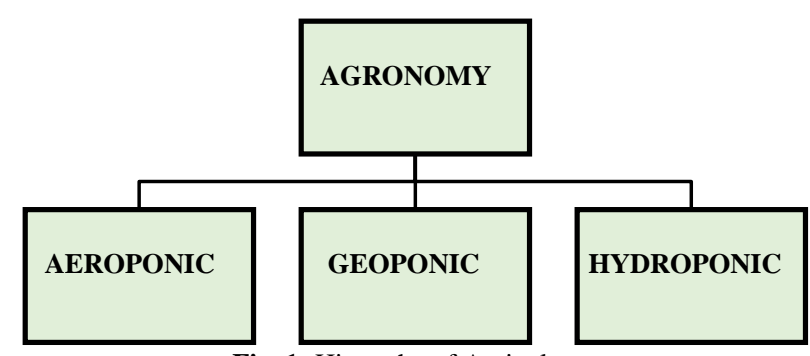

Fig. 1: Hierarchy of Agriculture.

\section{Image processing}

Image processing is the area, which deals with the processing of different images by means of a computer to get the desired results. The various steps involved in image processing, shown in Figure 2 follows.

\subsection{Image acquisition}

Image acquisition is theprocess of restoring an image from some origin e.g., a hardware based resource.

\subsection{Pre-processing}

The image preprocessing aims at correcting the degree of degradation in an image with the use of some specified steps. For this purpose, the prior information is necessary. It is performed mainly to increase the reliability of the data.

\subsection{Feature extraction}

It is the process of finding the reduced set of data of a given large data set. The reduced data set obtained is having all the feature of the initial data set. The various feature extraction techniques followed are Principal component analysis, deep feature synthesis, latent semantic analysis, edge detection, blob detection etc.

\subsection{Classification}

The classification is mainly done on the basis of supervised and unsupervised learning. Generally fuzzy logic, artificial neural network and chain codes are used for this process 


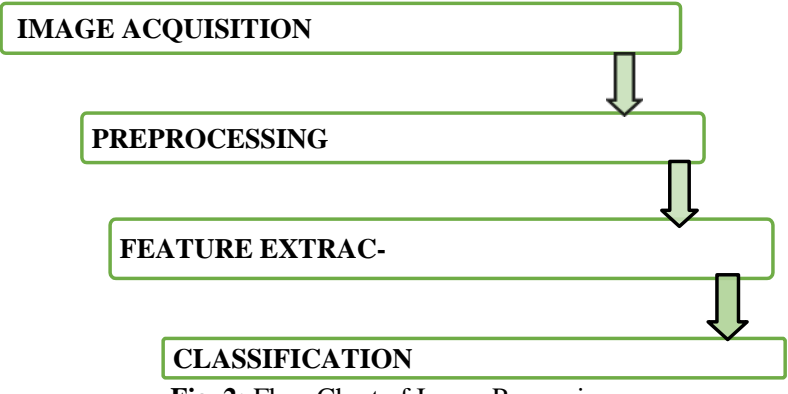

\section{Literature review}

Mrunmayee et al [1] present a diagnostic theory of Pomegranate plant diseases using the neural networks. In their paper they deal with the issues of plant pathology i.e., disease detection and classi fication. The diseases which they took into consideration were Bacterial Blight, Fruit Spot, Fruit Rot and Leaf Spot. k- means clustering technique was used for segmentation in which the distance between two pixels was measured by squared Euclidean distance. Feature extraction was done by using Gray Level Co-occurrence Matrix (GLCM) for obtaining the statistical texture features namely energy, covariance, sum entropy, difference entropy, entropy, information measure of correlation, inverse difference and contrast. By obtaining these results, a Multilayer Perceptrons (MLP) using backpropagation algorithm for the images was used in a neural network. A back-propagation algorithm with three phases' namely-training phase, recall phase and generalization phase was inducedPranjali et al [2] worked on the most widely grown fruit crop of India i.e., Grape. In their study they took the grape leaves into consideration. They focused on the bacterial, fungal and viral causes of the disease e.g., Powdery Mildew and Downy Mildew. The detection and classification of grape leaf disease was done. The given image is resized to size $300 * 300$ and then the thresholding is done to get all green color components. K-means algorithm is used in segmentation. For feature extraction the main type of features taken into consideration are shape, color and texture. First RGB was converted into HSV and then the image was subdivided into blocks and then mean, block variance and skewness is computed. For classification the Linear Support Vector Machine (LSVM) is used. P. Revathi et al [3] proposed an edge detection technique method for classifying cotton leaf diseases. Foliar the fungal disease was studied. HPCCD proposed algorithm is used to detect and categorize the disease. Colored image segmentation is done using RGB color models. Filtered data is then classified to identify the disease spots.Jun et al [4] gave a $n$ automatic segmentation method to detect the defects on the citrus surface eroded by diseases using circulatory threshold segmentation. The erosion coefficient is used to quantify the erosion degree. A comparative approach of performance of circulatory threshold Segmentation with respect to the Ostu segmentation method is proposed.Swati et al [5] used support vector machine and chain code to detect and classify the apple fruit diseases. They worked on scrub apple and bitter rot like diseases. For segmentation, the morphological operations were used. Multi-class support vector machine was used for classification.Ashwani et al [6] introduced fruit disease detection technique using color, texture analysis and artificial neural network. K-means clustering was used for segmentation and Open$\mathrm{CV}$ library was used in implementation. Artificial neural network concept was used in pattern matching and classification.Muhammad et al [7] implemented fuzzy logic and image processing in the orchid disease detection. The leaves were preprocessed using gray-scaling, threshold segmentation and noise removal. Fuzzification, fuzzy inference and defuzzification is used to find the results.Shiv et al [8] detected and classified apple fruit diseases using complete binary patterns. K-means clustering method is used in segmentation, and after feature extraction the Multi-class support vector machine is used for classification. Manish et al[9[ proposed pomegranate disease detection using image processing a web based tool is provided to identify the fruit disease. Feature extraction and clustering is done by k-means algorithm. Support vector machine which is used for classification.

\section{Pre-post processing approach analysis}

The use of different approaches by different authors so far is discussed in table. 1 and figure. 3 as follows.

\subsection{Image segmentation}

It is the process of portioning the digital image into different portions, such that the given data bifurcated and the respective useful information can be obtained from the given large data set. With this property use, the meaningful analysis can be done due to simplification. In image segmentation the labeling of the pixels is done so that the same type of pixels will share the same characteristics and thus can be grouped together and analysis becomes easy. Image segmentation can mostly be performed on the basis of color, texture or intensity.

\subsection{Feature extraction}

It is the process of building the informative and non-redundant extracted data set from the initial values of the given data set. It is mainly the process of dimensionality reduction. The features thus extracted are expected to contain the relevant information related to the given initial data set.

\subsection{Classification}

In machine learning and statistics, classification is the process of observing the new categories on the basis of observed sets, to which training set of data is formed. Classification depending on the nature of training data set can be differentiated as supervised and unsupervised learning. An algorithm that implements concrete classification is known as classifier.

Table 1: Tabular Representation of Different Approaches Used So Far

\begin{tabular}{|c|c|c|c|c|}
\hline S. no & Author Name & Segmentation Approach & Feature Extraction & Classifier \\
\hline 1 & $\begin{array}{l}\text { Jun Lu et al } \\
\text { [4] }\end{array}$ & Circularity gradient method & $\begin{array}{l}\text { B component of RGB color model is used to } \\
\text { Extract features. }\end{array}$ & No classifier used \\
\hline 2 & $\begin{array}{l}\text { Mrunmaye et } \\
\text { al [1] }\end{array}$ & k- means clustering algorithm & GLCM method & Artificial neural networks \\
\hline 3 & $\begin{array}{l}\text { Pranjali et al } \\
\text { [2] }\end{array}$ & k- means clustering algorithm & Color features are extracted & Support Vector Machines (SVM). \\
\hline 4 & $\begin{array}{l}\text { Swati et al } \\
\text { [5] }\end{array}$ & Morphological techniques & $\begin{array}{l}\text { Color, shape, texture and energy features } \\
\text { areinvolved in feature extraction. }\end{array}$ & $\begin{array}{l}\text { Support Vector Machines (SVM) } \\
\text { With radial kernel. }\end{array}$ \\
\hline 5 & $\begin{array}{l}\text { Ashvani et al } \\
\text { [6] }\end{array}$ & k-means clustering algorithm & SURF algorithm & $\begin{array}{l}\text { Mathematical model is used for } \\
\text { classification }\end{array}$ \\
\hline 6 & $\begin{array}{l}\text { Shiv Ram et } \\
\text { al [8] }\end{array}$ & k-means clustering algorithm & $\begin{array}{l}\text { Feature extraction based on the Global Color } \\
\text { Histogram (GCH), Color Coherence Vector } \\
\text { (CCV), Local Binary Pattern (LBP) is } \\
\text { performed }\end{array}$ & $\begin{array}{l}\text { Multi-class Support Vector } \\
\text { Machine (MVSM). }\end{array}$ \\
\hline 7 & Muhammd et & Image gray scaling and & Ostu method & Membership functions are used. \\
\hline
\end{tabular}


al [7] determining $\quad$ Fuzzy inference is used.

Defuzzification method is

implemented.

8 Manisha et k-means clustering algorithm Color, morphology and CCV.

Support Vector Machines (SVM).
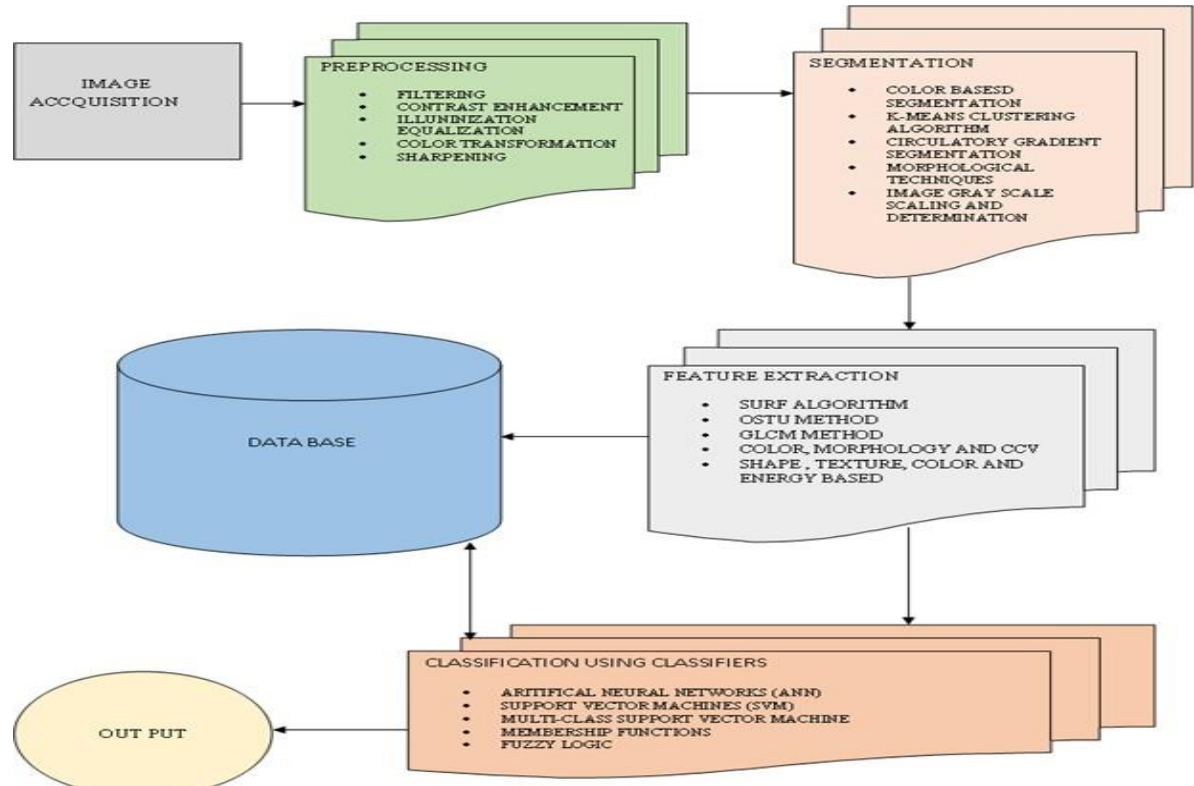

Fig. 3: Block Diagram of Fruit Disease Detection Using Image Processing.

\section{Review of different fruit detection methodol- ogies}

Table 2: Review of Different Fruit Detection Methodologies

\begin{tabular}{l} 
Author Title \\
\hline \\
Jun Lu et $\begin{array}{l}\text { Detecting Defects on Citrus } \\
\text { al [4] }\end{array}$
\end{tabular}

al [4] Threshold Segmentation

Set

500

data

sets.
Pranjali SVM Classifier Based Grape et al [2] Leaf Disease Detection Set Methodology

\section{Conclusion}

- "Automatic segmentation" method proposed to detect defects on citrus surface eroded by diseases and pests based on "circularity threshold" segmentation.

- "Erosion coefficient "is used to quantify the erosion degree.

- "Circularity gradient "segmentation is used.

$15.58 \%$ improvement using circularity

- Segmentation approach used was compared to "Ostu seg- gradient method with respect to Global

maye et Plant Diseases using Neura

al [1] network
137

data

sets
Detection and Classification for Swati et apple fruit diseases using sup- 300 al [5] port vector machine and chain $\begin{aligned} & \text { data } \\ & \text { sets }\end{aligned}$ code. mentation" method for the performance check.

- "RGB color" model used for finding a contrast between background and the fruits and most obvious results were found in the "B component"

- Map in the RGB color model.

- Training and testing of the images is done.

- The color images are preprocessed using the filters and then undergo $\mathrm{k}$-means clustering segmentation.

- GLCM method is used to extract texture features and thus the results are given to the artificial neural network

- Preprocessing of the images is done by resizing, thresholding, and Gaussian filtering.

Otsu method

$\mathrm{K}$-means clustering algorithm is used for segmentation. Texture and color features are used for feature extraction. Image feature database is classified using the support vector machines

- $\quad(\mathrm{SVM})$

- Worked mainly on three data sets of apple namely- normal apple, scrub apple, bitter rot apple.

- Data preprocessing is done.

- $\quad$ RGB to Gray conversion, median filter, edge detector andSupport vector machine with radial basis morphology techniques are used for image segmenta- kernel achieves $98 \%$ classification accution. Color, shape, texture and energy features are in- racy. volved in feature extraction.

- Chain code is prepared on the basis of the outer edge of the object and travelling direction.
- Good fruit and good leaf gives $100 \%$ result.

- Leaf spot gives $87.50 \%$. Bacterial blight gives $85.71 \%$.

- White fruit spot and fruit rot gives $83.33 \%$ results

- $90 \%$.

- The results based on only on texture are $100 \%$.

- $\quad$ Texture + color results are $88.89 \%$ accurate.
- The overall accuracy is 
- Multi class support vector machines with radial kernel support Training and classification.

- Diagnose and classify external disease within fruits.

- Open CV library used for implementation. K- means clustering algorithm is used for segmentation.

- Trained database of infected image is generated using artificial neural network.

Ashvani Fruit Disease Detection using

et al [6] Color, Texture Analysis and n/a ANN
Manisha Smart Farming:

et al [9] Pomegranate Disease Detection Using Image Processing.
- SURF algorithm is used as locator and descriptor for extracting the features based on the color, morphology, structure and texture.

- Blob Analysis is used to determine the scope of interest.

- Pattern matching is applied on the basis of the concept of artificial neural network.

- Mathematical model is given for feature

- Extraction of image classification.

- $\quad$ - Morphological filter is used for the noise

- Removal.

COA:-

- $\quad$ - The data after preprocessing is acquired by

- Using the image processing toolbox in MATLAB.

- - For implementing the fuzzy logic, the

- Membership functions are used.

- - Fuzzy inference is done

- - Defuzzification method e.g., Centre of

- Maximum (COM), Mean of Maximum (MOM),

- and the Centre of Area (COA) are used to

- translate the linguistic value back into crisp

- Value

- - Web based tool is provided for disease

- Detection

- - K-means clustering is used for segmentation.

- - Feature extraction on the basis of color,

- Morphology and CCV.

- - SVM is used for classification.
A solution is proposed for the grape, apple and pomegranate fruit disease detection with minor computational effort.
$0.399-0.729$

For Bacterial Blight the

Overall accuracy is $82 \%$.

\section{Conclusion}

Based on the different diseases, the different approaches were used by authors to detect the fruit diseases under image processing. The focus should be on how to collect or take the images. The images should be taken under the precise and static environment so that the accuracy and performance thus obtained is vital and viable. Through this paper, we have studied different approaches and their accuracy and performance level. According to our study in image segmentation, the $\mathrm{k}$-means clustering algorithm is most prominently used due to ease and accuracy, while in case of classifiers; the SVM classifiers have shown more accuracy and usage. Under this area more futuristic work can be performed as there is lot of scope under various parameters in this area which have not been taken under study till now.

\section{References}

[1] MrunmayeeDhakate and Ingole A.B, "Diagnosis of Pomegranate Plant Diseases using Neural Network", Fifth National Conference on Computer Vision, Pattern Recognition, Image Processing and Graphics (NCVPRIPG), PP: 1-4, 16-19 Dec. 2015.

[2] PranjaliB.Padol and Prof. Anjali A.Yadav, "SVM Classifier Based Grape Leaf Disease Detection", Conference on Advances in Signal Processing (CASP), PP: 175 - 179, 9-11 June 2016.

[3] P.Revathi and M.Hemalatha, "Classification of Cotton Leaf Spot Diseases Using Image Processing Edge Detection Techniques", International Conference on Emerging Trends in Science, Engineering and Technology (INCOSET), PP: 169 - 173, 13-14 Dec. 2012.

[4] Jun Lu, Pengfei Wu, JiweiXue, Ming Qiu and Fan Peng, "Detecting Defects on Citrus Surface Based on Circularity Threshold Segmentation", 12th International Conference on Fuzzy Systems and Knowledge Discovery (FSKD), PP: 1543 - 1547, 15-17 Aug. 2015

[5] Swati Dewliya and Pratibha Singh, "Detection and classification for apple fruit diseases using support vector machine and chain code", International Research Journal of Engineering and Technology (IRJET), Volume: 02 Issue: 04-Aug-2015.

[6] AshwaniAwate, DaminiDeshmankar, GayatriAmrutkar, UtkarshaBagul and SamdhanSonavane, "Fruit Disease Detection using
Color, Texture Analysis and ANN", International Conference on Green Computing and Internet of Things (ICGCIoT), PP: 970 - 975, 8-10 Oct. 2015.

[7] Muhammad Thaqif bin Mohamad Azmi and Naimah Mat Isa, "Orchid Disease Detection Using Image Processing and Fuzzy Logic", International Conference on Electrical, Electronics and System Engineering (ICEESE), PP: 37 - 42, 4-5 Dec. 2013.

[8] Shiv Ram Dubey and Anand Singh Jalal, "Detection and Classification of Apple Fruit Diseases Using Complete Local Binary Patterns", Third International Conference on Computer and Communication Technology (ICCCT), PP: 346-351, 23-25 Nov. 2012.

[9] ManishaBhange and H.A. Hingoliwala, "Smart Farming: Pomegranate Disease Detection Using Image Processing", Second International Symposium on Computer Vision and the Internet (VisionNet'15), PP 280-288, 22 Aug. 2015.

[10] Mendoza, O.; Melin, Patricia; Licea, G., "A New Method for Edge Detection in Image Processing Using Interval Type-2 Fuzzy Logic," Granular Computing, 2007. GRC 2007. IEEE International Conference, PP: 151-151, 2-4 Nov. 2007.

[11] Choudhary, G.K.; Dey, S., "Crack detection in concrete surfaces using image processing, fuzzy logic, and neural networks," Advanced Computational Intelligence (ICACI), 2012 IEEE Fifth International Conference, and PP: 404-411, 18-20 Oct. 2012.

[12] Nithiya, N.; Imtiaz, R., "Fuzzy logic-based implementation of color image processing techniques in FPGA," Information Communication and Embedded Systems (ICICES), 2013 International Conference, PP: 1114-1117, 21-22 Feb. 2013.

[13] J. Blasco, N. Aleixos, J. Gomez, E. Molto, "Citrus sorting by identification of the most common defects using multispectral computer vision", Journal of Food Engineering. 2007, PP: 384- 393, 2007.

[14] Wen Zhiyuan, Cao Leping, "Machine Identification of Disease and Insect Pest of Citrus Fruit" in Chinese Journal of Engineering Mathematics, Chinese with English abstract, PP: 41-646, 2012.

[15] P. Chaudhary, A. K. Chaudhari, A. N. Cheeran, S. Godara, "Color Transform Based Approach for Disease Spot", International Journal of Computer Science and Telecommunications, PP: 65-70, June 2012.

[16] Tejal Deshpande, SharmilaSengupta, and K.S.Raghuvanshi, "Grading identification of disease in pomegranate leaf and fruit", International Journal of Computer Science and Information Technologies, PP: 4638-4645, August 2014. 
[17] TimoOjala, MattiPietikainen, and TopiMaenpaa, "Multiresolution grayscale and rotation invariant texture classification with local binary pattern", IEEE Trans. On Pattern Analysis and Machine Intelligence, PP: 971-987, 2002.

[18] Simon Haykins, "An Introduction to Artificial Neural Networks", Pearson Publications, 2005.

[19] PradnyaRavindraNarvekar, Mahesh ManikKumbhar, and S. N. Patil, "Grape leaf diseases detection analysis using sgdm matrix method", International Journal of Innovative Research inComputer and Communication Engineering, PP: 287-291, March 2014.

[20] DaeGwan Kim, Thomas F. Burks, and Duke M. BulanonJianwei feature analysis", International Journal on Agriculture and Biological Engineering, PP: 41-50, Sept 2009. 